\title{
openheart Health-related quality of life and lived experiences in males and females with thoracic aortic disease and their partners
}

\author{
Carlijn G E Thijssen, ${ }^{1,2}$ Silvy Dekker, ${ }^{1}$ Lidia Rianne Bons, ${ }^{1}$ Arjen L Gökalp, ${ }^{3}$ \\ Robert M Kauling, ${ }^{1}$ Annemien E van den Bosch, ${ }^{1}$ \\ Judith Anne Adriane Ellen Cuypers, ${ }^{1}$ Elisabeth M W J Utens, ${ }^{4,5}$ \\ Roland R L van Kimmenade, ${ }^{1,2}$ Johanna J M Takkenberg, ${ }^{3}$ Leontien M H Roos, ${ }^{6}$ \\ Eva Goossens (D) , 7,8,9 Jolien W Roos-Hesselink (D) ${ }^{1}$
}

To cite: Thijssen CGE, Dekker S, Bons LR, et al. Health-related quality of life and lived experiences in males and females with thoracic aortic disease and their partners. Open Heart 2020;7:e001419. doi:10.1136/ openhrt-2020-001419

CGET and SD contributed equally.

EG and JWR-H contributed equally.

Received 14 August 2020 Revised 3 September 2020 Accepted 3 September 2020

Check for updates

(c) Author(s) (or their employer(s)) 2020. Re-use permitted under CC BY-NC. No commercial re-use. See rights and permissions. Published by BMJ.

For numbered affiliations see end of article.

Correspondence to Dr Jolien W Roos-Hesselink; j. roos@erasmusmc.nl

\section{ABSTRACT}

Objective Thoracic aortic disease (TAD) may have substantial impact on health-related quality of life (HRQOL). We described HRQOL in patients with TAD, cardiovascular screening participants and their partners; identified factors associated with HRQOL; and explored lived experiences and feelings of anxiety or depression using a mixed methods design.

Methods For this cross-sectional study, all consecutive patients visiting the TAD outpatient clinic (2017-2019) at our centre were asked to complete three questionnaires: the Short Form 36 (SF-36), the Hospital Anxiety and Depression Scale (HADS) and the Rotterdam Disease Specific Questionnaire (RDSQ). A subsample was invited for in-depth interviews.

Results In total, 261 participants were included: 147 patients with TAD (thoracic aortic diameter $\geq 40$ $\mathrm{mm}$; 54 females, $36.7 \%)$, 114 screening participants (cardiovascular family screening; 71 females, 62.3\%) and 66 partners. Compared with the general population, patients with TAD showed markedly lower HRQOL, whereas screening participants' HRQOL was less impaired. Female and younger participants scored significantly lower on the SF-36 and HADS compared with male and older participants. Smaller aortic diameter was associated with better RDSQ score, and previous aortic surgery was associated with higher HADS depression scores. Furthermore, partners scored significantly lower on 2/8 SF-36 subdomains when compared with the general population. From 11 interviewees, determinants of psychological distress included coping strategies, impact on social and professional life, disease-related knowledge, state of aortic diameters and physical symptoms.

Conclusions Healthcare professionals must be aware of HRQOL impairments in patients with TAD, particularly in younger females. Moreover, attention for partners is needed. Coping strategies and communication within the family were found to be important factors influencing psychological distress, and might be valuable leads for counselling and HRQOL improvement in this population.

\section{INTRODUCTION}

Thoracic aortic disease (TAD), including thoracic aortic aneurysms and dissections, has

\section{Key questions}

What is already known about this subject?

- Health-related quality of life (HRQOL) is known to be reduced in patients with thoracic aortic disease (TAD).

- Male-female-specific HRQOL, partners' HRQOL and HRQOL of individuals who undergo family screening for TAD have not yet been investigated.

- Combined qualitative and quantitative (ie, mixed methods) research has been suggested to gain more specific insights into the impact of TAD on HRQOL.

What does this study add?

- This study showed that HRQOL in screening participants is affected to a lesser extent compared with patients with confirmed thoracic aortic aneurysm. Especially female and younger patients showed Iower HRQOL.

- Partners' HRQOL was reduced compared with the general population.

- Thoracic aortic diameter and thoracic aortic surgery were found to be important factors, which negatively influenced HRQOL.

- Interviewed patients experienced variable levels of psychological distress, which were triggered by the evolution of the aortic disease, level of diseaserelated knowledge, loss of identity, sports participation and vocational choice, physical complaints and communication within their family.

How might this impact on clinical practice?

- To provide the necessary guidance and counselling to patients, healthcare workers need to be aware of the significant HRQOL impairments and psychological distress in this patient population, particularly in younger female patients.

- Adequate coping strategies and good communication styles within the family seem to be important factors for influencing psychological distress.

- In addition, more attention for partners of patients with TAD is clearly warranted. 
an estimated prevalence of 9/100 000 per year in females, and 16/100 000 per year in males. ${ }^{1}$ These patients are at risk of sudden cardiac death. ${ }^{2}$ An inherited pattern of TAD is found in about $20 \%$ of cases. ${ }^{3}$ Therefore, patients and family members are often included in genetic and cardiovascular screening programmes, and may face lifelong uncertainties. ${ }^{4}$

The scarce evidence on health-related quality of life (HRQOL) in patients with TAD showed suboptimal results. ${ }^{56}$ Most research has been performed in patients with hereditary thoracic aortic diseases (HTAD) such as Marfan syndrome and Loeys-Dietz syndrome. In patients with HTAD, HRQOL was reduced as compared with the general population, and comparable to other chronic diseases. $^{7-10}$

To achieve HRQOL improvement, it is important to obtain more patient and disease-specific information on HRQOL and lived experiences. However, no studies to date have evaluated male-female-specific HRQOL in patients with TAD. Furthermore, the impact of the disease on HRQOL of partners of patients with TAD has not yet been investigated. It has been suggested that studies using disease-specific questionnaires and a combined quantitative and qualitative approach would provide better insight. ${ }^{6}$ Therefore, in addition to existing well-validated questionnaires, we developed a disease-specific questionnaire, and aimed to: 1) evaluate HRQOL, anxiety and depression in patients with TAD, screening participants and partners and compare this with the general population, 2) compare HRQOL between males and females, 3) identify factors associated with HRQOL and 4) explore lived experiences of patients with TAD and their feelings of anxiety or depression.

\section{METHODS}

\section{Study populations}

All consecutive patients who visited the specialised TAD outpatient clinic of our tertiary care centre between October 2017 and July 2019, were eligible for inclusion. The TAD outpatient clinic is a specialised outpatient clinic in which patients are seen by cardiologists or physician assistants for cardiovascular family screening or aortic surveillance, including referral from primary care and secondary care. Inclusion criteria were: adult age $(\geq 18$ years), outpatient clinic visit for cardiovascular (family) screening or follow-up of TAD. Exclusion criteria were intellectual disability or language barrier. All eligible patients were invited to participate in the study, reasons for exclusion or failure to complete the questionnaires are shown in figure 1 . Included participants were divided into two groups: participants with a (genetic) predisposition and/or positive family history of TAD, hereafter referred to as 'screening participants'; and 'patients with TAD' under surveillance for confirmed thoracic aortic aneurysm defined as a thoracic aortic diameter of $\geq 40$ $\mathrm{mm}$.
FIGURE 1. Flowchart of patient inclusion

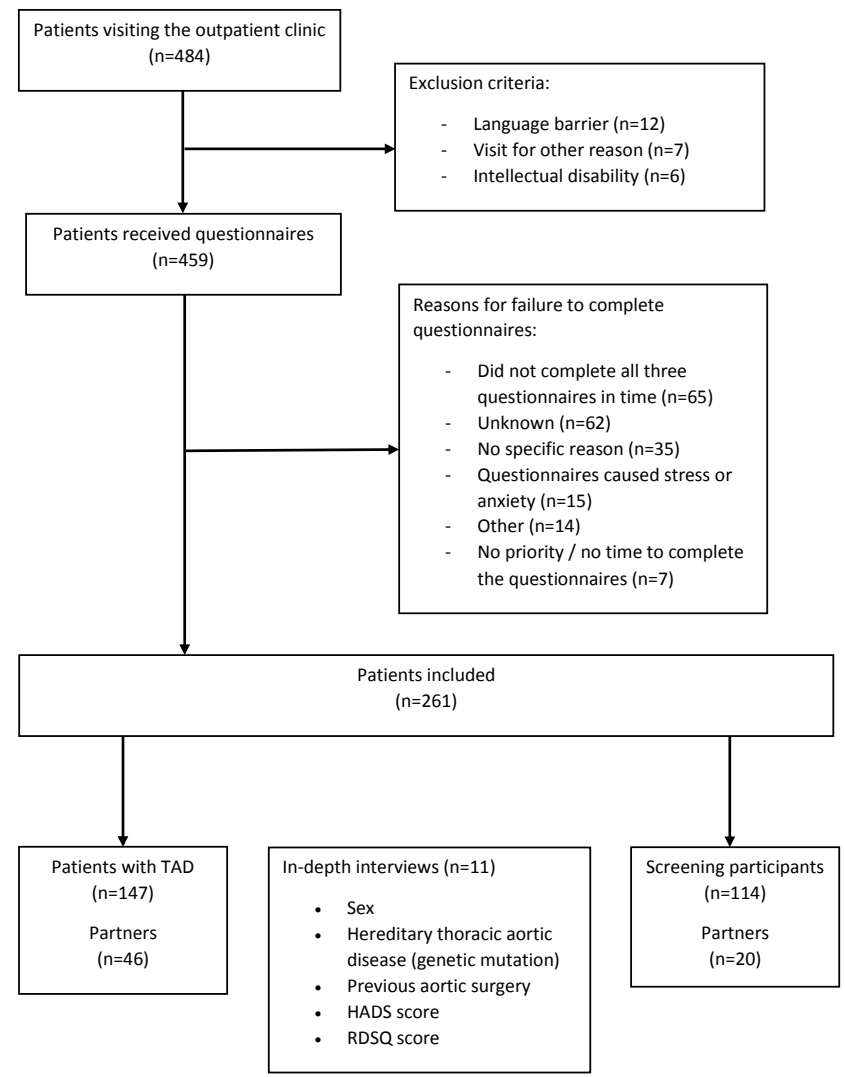

TAD patients= Patients with confirmed thoracic aortic aneurysm $\geq 40 \mathrm{~mm}$; HADS = Hospital Anxiety and Depression Scale; RDSQ= Rotterdam Disease Specific Questionnaire.

Figure 1 Flow chart of patient inclusion. HADS, Hospital Anxiety and Depression Scale; RDSQ, Rotterdam Disease Specific Questionnaire; TAD, thoracic aortic aneurysm $\geq 40$ $\mathrm{mm}$.

\section{Data collection}

All included patients completed three questionnaires: the Short Form 36 (SF-36), the Hospital Anxiety and Depression Scale (HADS) and the Rotterdam Disease Specific Questionnaire for TAD (RDSQ), which was developed for this study. The questionnaires were distributed 1-2 weeks before the scheduled outpatient visit. Partners were asked to complete the same set of questionnaires either during the outpatient clinic visit or via email. All questionnaires were documented using a secured web-based application for distribution of questionnaires during clinical research (GEneric Medical Survey Tracker, Erasmus MC and Equipe Zorgbedrijven, latest release 2019, V.1.8.6, open source). This application did not allow any missing answers. Patients who did not complete all three questionnaires were excluded. Additional data were collected from the patient files using a standardised case report form. Body surface area (BSA) was calculated using the DuBois and DuBois formula. ${ }^{11}$ 


\section{Questionnaires}

The SF-36 questionnaire is a widely used HRQOL questionnaire with 36 items, which has eight domains: Physical Functioning (PF), Role limitations due to Physical health problems (RP), Bodily Pain (BP), General Health perceptions (GH), Vitality (VT), Social Functioning (SF), Role limitations due to Emotional problems (RE) and general Mental Health (psychological distress) (MH). ${ }^{12} 13$ The first four domains (PF, RP, BP and $\mathrm{GH}$ ) together form the Physical Component Summary (PCS) and the last four domains (VT, SF, RE and MH) form the Mental Component Summary (MCS). All SF-36 subdomains have a score range of $0-100$, with higher scores reflecting a better quality of life. The SF-36 has been translated and validated in the Dutch language. ${ }^{12}$ Male and female SF-36 scores of participants were compared with male-femalespecific norm values. ${ }^{12}$ Unfortunately, no age-specific norm values were available for males and females separately. Therefore, an additional analysis was performed to compare participants with an age-matched general Dutch population. $^{12}$ Likewise, SF-36 scores of partners were compared with the general population. Male-femalespecific PCS and MCS were calculated using the mean and SD of the general Dutch population.

The HADS questionnaire assesses signs of anxiety and depression, and has been validated in the Dutch language. ${ }^{14}$ This 14 -item scale is divided in two dimensions: anxiety (seven items) and depression (seven items). The responses result in a score for each dimension with a score range of 0-21 and a total overall score with a score range of 0-42. Higher scores represent higher levels of anxiety and depression. A score of $\geq 8$ on the HADS anxiety and depression subscales is internationally used as the cut-off score to define elevated levels of anxiety/ depression. ${ }^{15}$ HADS scores of participants were compared with age-matched norm values. ${ }^{14}$

The RDSQ for TAD was developed by a multidisciplinary team in our centre, including a cardiologist, psychologist, PhD candidate and physician assistant (online supplemental file 1). ${ }^{10}$ The purpose of this disease-specific questionnaire was to assess the impact of having TAD on daily life factors, such as: employment; family life; sexual functioning and sports participation. The questionnaire contains 18 statements which participants were asked to rate on a 10-point Likert scale. A higher score reflects better agreement with the statements, and more impaired TAD-related quality of life.

\section{In-depth interviews}

Eleven participants were purposively selected by an independent researcher based on differences in age, sex, genetic disorder, previous surgery, symptoms of depression and anxiety (HADS score) and RDSQ score (figure 1), in order to create a heterogeneous group. They were invited for a semi-structured qualitative interview using a topic list, which evolved over the course of the study as an iterative process. Interviews were audiotaped using a voice recorder and non-verbal signs were noted. A verbatim transcript of the interviews was made on the same day.

The verbatim transcripts were analysed using content analysis, applying a multistep consecutive approach starting with an initial open coding phase, followed by axial coding and finishing with a selective coding process. ${ }^{17}$ In addition to the interviewer (SD), two other researchers (CGET and EG) read the interview transcripts, and the individual narrative of each participant was constructed (ie, open coding). In the second phase, these researchers independently coded the individual narratives to identify significant and common aspects (ie, axial coding). When necessary, decisions were made in consensus. In a third phase, SD and EG analysed the codes together to construct the narrative syntheses (ie, selective coding) and the final coding tree, including an overview of the most important factors. The coding process was carried out using the NVIVO V.12 plus software (QSR International, March 2018).

\section{Statistical analysis}

Data were analysed using SPSS statistics (IBM SPSS Statistics V.25). Continuous data were presented as mean and SD when normally distributed, and as median with IQR when skewed. Categorical data were presented as frequencies with percentages. Comparison between patient and normative data was analysed using means and SD. Unpaired Student's t-test or non-parametric Wilcoxon test were used to compare continuous variables, $\chi^{2}$ test or Fisher's exact test were used to compare categorical variables. The one-sample Student's t-test was used to compare norm-values with the study population means. Associations between baseline characteristics and RDSQ and HADS scores were evaluated using univariable linear regression models. Factors with a $\mathrm{p}$ value of $<0.2$ and factors that were considered clinically relevant were considered for multivariable linear regression models. All models were adjusted for age and sex. Linear regression analyses were performed for screening participants and patients with TAD combined, and for partners separately. An $\alpha$-value of $<0.05$ was considered significant. Bonferroni correction for multiple testing was applied.

\section{RESULTS}

\section{Patient population}

Figure 1 shows a flow chart of the inclusion process. In total, 261 participants were included comprising 147 patients with TAD (54 females, $36.7 \%$ ) and 114 screening participants (71 females, 62.3\%). Furthermore, 66 partners completed the questionnaires. The partners' sex was registered in 53 participants $(80 \%)$, of which $63 \%$ was female.

Table 1 shows baseline characteristics of included males and females. Males showed significantly higher height, weight, BSA and absolute diameters of the thoracic aorta. Females showed higher adjusted ascending aortic diameters (indexed for BSA). A significantly higher percentage 
Table 1 Patient characteristics

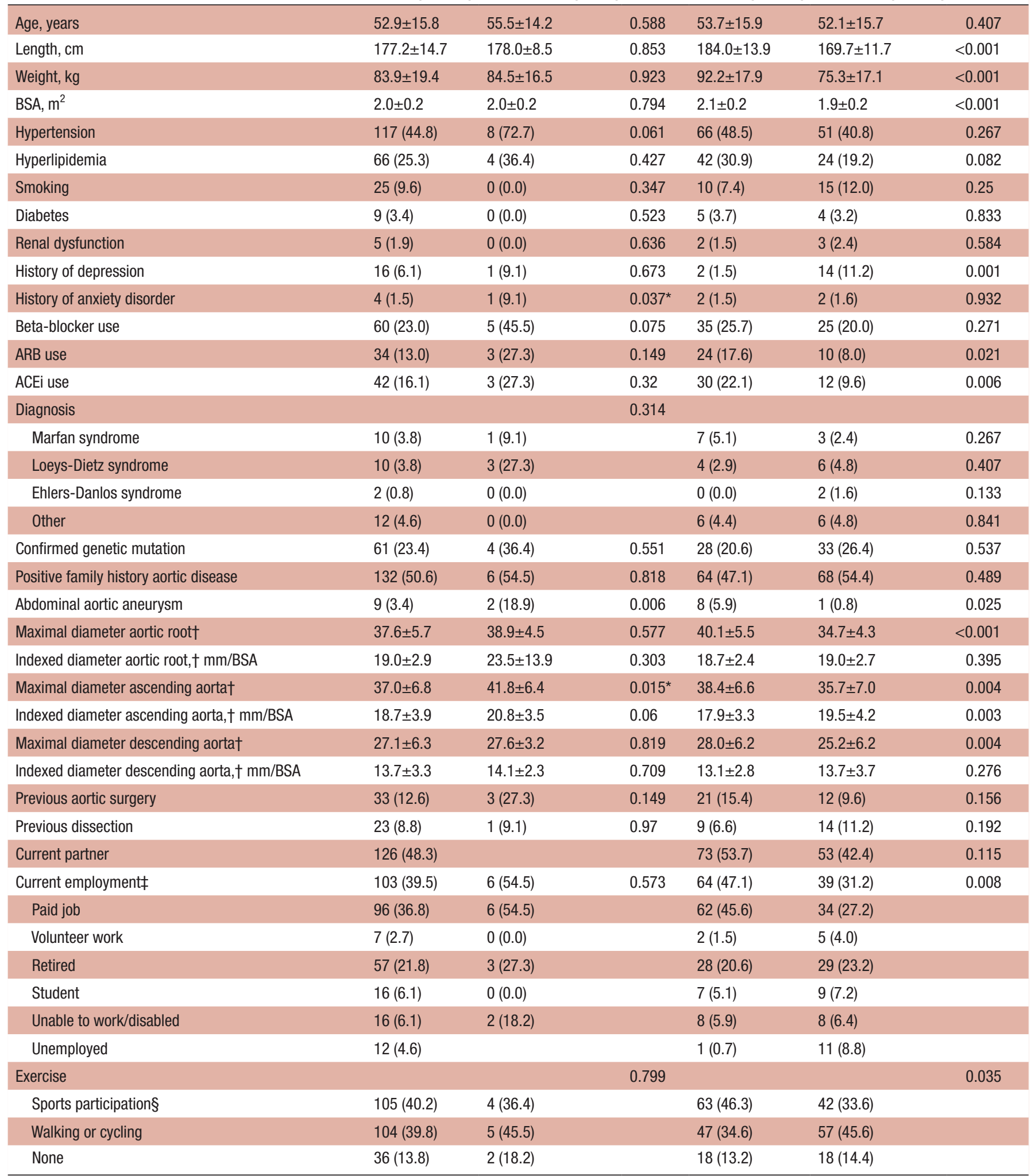

Data are expressed as mean \pm SD or as absolute and percentage.

*Only patients without previous aortic surgery.

†Current employment=paid job or volunteer work.

‡Defined as: participating in any sport other than daily walking or cycling at any level at least once a week.

ACEi, ACE inhibitor; ARB, angiotensin II receptor blocker; BSA, body surface area. 
FIGURE 2. Comparison of SF-36 scores of male and female participants to the general population.
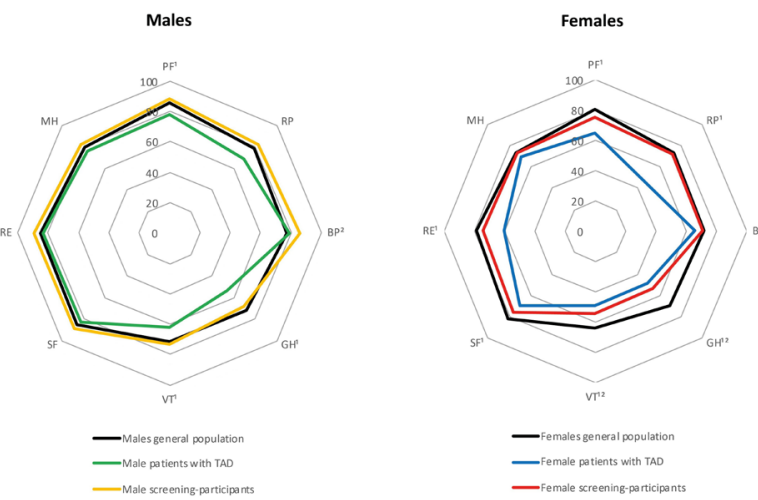

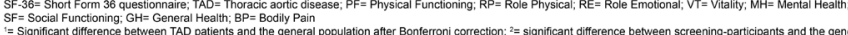

Figure 2 Comparison of SF-36 scores of male and female participants with the general population. ${ }^{1}$ Significant difference between patients with TAD and the general population after Bonferroni correction. ${ }^{2}$ Significant difference between screening participants and the general population after Bonferroni correction. BP, Bodily Pain; GH, General Health; MH, Mental Health; PF, Physical Functioning; RP, Role Physical; RE, Role Emotional; SF-36, Short Form 36 questionnaire; SF, Social Functioning; TAD, thoracic aortic disease; VT, Vitality.

of males used ACE inhibitors $(p=0.006)$ and angiotensin receptor blockers (ARBs) $(p=0.021)$. Males were more often employed, and performed regular exercise more often, whereas females more often had a history of depression. Additionally, baseline characteristics between patients with TAD and screening participants were compared (online supplemental file 2 ).

\section{Scores of patients with TAD and screening participants compared with the general population}

Figure 2 shows comparison of SF-36 subscores of patients with TAD and screening participants with a sex-matched general population. Male screening participants showed a significantly higher score on the subdomain BP $(77.3 \pm 22.7$ vs $86.3 \pm 19.5, \mathrm{p}=0.004$ ), whereas male patients with TAD showed significantly lower scores on PF $(77.9 \pm 25.0$ vs 85.4 $\pm 21.0, \mathrm{p}=0.005), \mathrm{GH}(54.0 \pm 23.1$ vs $71.6 \pm 20.6, \mathrm{p}<0.001)$ and VT $(61.7 \pm 24.1$ vs $71.9 \pm 18.3$, $\mathrm{p}<0.001)$. Female screening participants showed a significantly lower score on GH $(54.0 \pm 25.8$ vs $69.9 \pm 20.6, \mathrm{p}<0.001)$ and VT $(54.8 \pm 26.4$ vs $64.3 \pm 19.7, \mathrm{p}=0.003)$. Female patients with TAD showed significantly lower scores on all SF-36 subdomains except BP and $\mathrm{MH}$ when compared with the general population: $\mathrm{PF}$ $64.7 \pm 26.3$ vs $80.4 \pm 24.2, p \leq 0.001$; RP $46.3 \pm 42.5$ vs $73.8 \pm 38.5$, $\mathrm{p} \leq 0.001 ;$ GH $49.0 \pm 21.9$ vs $69.9 \pm 20.6$, $\mathrm{p} \leq 0.001 ; \mathrm{VT} 49.0 \pm 23.6$ vs $64.3 \pm 19.7, \mathrm{p} \leq 0.001$; $\mathrm{SF} 69.9 \pm 28.8$ vs $82.0 \pm 23.5, \mathrm{p}=0.003$; RE $60.5 \pm 42.0$ vs $78.5 \pm 35.7, p=0.003$.

Figure 3 shows SF-36 scores of screening participants and patients with TAD compared with the age-matched general population. Younger patients with TAD and screening participants showed lower scores on all SF-36 subdomains, with increasing age this difference became smaller. HADS scores of patients with TAD and screening participants

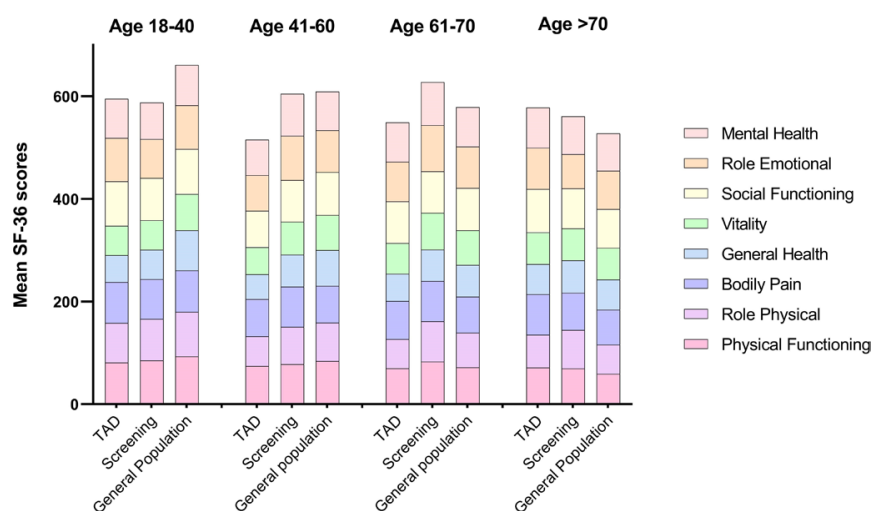

Figure 3 Comparison of SF-36 scores of participants with age-matched general population. TAD, thoracic aortic disease, defined as confirmed thoracic aortic aneurysm of $\geq$ 40 mm; SF-36, Short Form 36 questionnaire.

aged 18-65 years were not significantly different compared with the age-matched general population. Patients with TAD and screening participants older than 65 years showed lower scores when compared with the age-matched general population, especially on the HADS depression subdomain $(2.9 \pm 3.7$ vs $4.6 \pm 3.6, \mathrm{p}=0.001)$.

\section{Differences between HRQOL scores of males and females}

Table 2 shows scores of male and female participants on subdomains of all questionnaires. Elevated scores $(\geq 8)$ on the HADS anxiety subdomain occurred significantly more often in females $(12.5 \%$ vs $29.6 \%, \mathrm{p}=0.001)$.

Online supplemental file 3 shows stratified analysis of male-female HRQOL for screening participants and patients with TAD. Results were comparable to the unstratified analysis.

Online supplemental file 4 shows median scores of males and females on the RDSQ questionnaire. A significant difference was found between males and females on question 13, which indicates whether the participant experiences more anxiety than before the (potential) diagnosis of TAD (1.0 (IQR 1.0-3.0) vs 2.0 (IQR 1.0-5.0), $\mathrm{p}=0.011$ ) and question 17 which indicates worries about the heredity of TAD (5.0 (IQR 2.0-8.0) vs 7.0 (IQR 5.0-9.0), p<0.001).

\section{Health-related quality of life of partners}

Table 3 shows the partners' scores on all three questionnaires. Scores in partners were not significantly different compared with patients with TAD and screening participants. When compared with the general population, partners showed significantly lower scores on 2/8 SF-36 subdomains after Bonferroni correction: GH and VT. There were no significant differences in scores on the three questionnaires between partners of patients with TAD $(n=46)$ and partners of screening participants $(n=20)$.

\section{Factors associated with HRQOL in patients with TAD, screening-participants and their partners}

Multivariable analysis showed only history of anxiety disorder was associated with higher HADS anxiety score. Additionally, history of anxiety disorder, previous aortic 


\begin{tabular}{|c|c|c|c|c|}
\hline & Total $(n=261)$ & Males $(n=136)$ & Females $(n=125)$ & $P$ value \\
\hline \multicolumn{5}{|l|}{ RDSQ } \\
\hline Total score & $51.0(32.0-80.0)$ & $48.0(29.3-78.5)$ & $53.0(34.5-85.5)$ & 0.160 \\
\hline \multicolumn{5}{|l|}{ SF-36 } \\
\hline Physical Functioning & $85.0(60.0-95.0)$ & $95.0(71.3-100.0)$ & $80.0(50.0-95.0)$ & $<0.001^{*}$ \\
\hline Role Physical & $100.0(25.0-100.0)$ & $100.0(50.0-100.0)$ & $75.0(12.5-100.0)$ & 0.024 \\
\hline Bodily Pain & $80.0(57.5-100.0)$ & $90.0(67.5-100.0)$ & $67.5(45.0-100.0)$ & $<0.001^{*}$ \\
\hline General Health & $55.0(40.0-100.0)$ & $60.0(40.0-80.0)$ & $55.0(35.0-70.0)$ & 0.030 \\
\hline Vitality & $60.0(40.0-80.0)$ & $70.0(50.0-83.8)$ & $50.0(35.0-75.0)$ & $<0.001^{*}$ \\
\hline Social Functioning & $87.5(62.5-100.0)$ & $100.0(75.0-100.0)$ & $75.0(62.5-100.0)$ & $<0.001^{*}$ \\
\hline Role Emotional & $100.0(66.7-100.0)$ & $100.0(100.0-100.0)$ & $100.0(33.3-100.0)$ & $<0.001^{*}$ \\
\hline Mental health & $80.0(64.0-88.0)$ & $84.0(69.0-92.0)$ & $76.0(56.0-88.0)$ & 0.007 \\
\hline PCS & $49.2(38.2-55.8)$ & $51.6(41.8-56.3)$ & $46.2(35.4-54.5)$ & 0.017 \\
\hline MCS & $51.9(42.8-56.9)$ & $54.4(48.0-57.4)$ & $48.5(37.9-55.0)$ & $<0.001^{*}$ \\
\hline \multicolumn{5}{|l|}{ HADS } \\
\hline Anxiety score & $4.0(2.0-7.0)$ & $4.0(2.0-6.0)$ & $4.0(2.0-8.0)$ & 0.023 \\
\hline Depression score & $2.0(1.0-5.0)$ & $1.5(0.0-4.8)$ & $2.0(1.0-5.0)$ & 0.043 \\
\hline Anxiety score $\geq 8$ & $54(20.7)$ & $17(12.5)$ & $37(29.6)$ & $0.001^{*}$ \\
\hline Depression score $\geq 8$ & $39(14.9)$ & $16(11.8)$ & $23(18.4)$ & 0.133 \\
\hline Total score & $6.0(3.0-12.0)$ & $5.0(2.3-10.0)$ & $6.0(4.0-15.5)$ & 0.037 \\
\hline
\end{tabular}

Data was non-parametrically distributed and therefore expressed as medians (IQR), or as absolute and percentage.

*Significant after Bonferroni correction.

HADS, Hospital Anxiety and Depression Scale; MCS, Mental Component Summary; Participants, screening participants and patients with thoracic aortic disease; PCS, Physical Component Summary; RDSQ, Rotterdam Disease Specific Questionnaire; SF-36, Short Form 36.

surgery, diabetes and indexed descending aortic diameter were found to be associated with higher HADS depression score. Higher RDSQ score was significantly associated with employment.

ARB use and history of aortic dissection in participants were associated with higher HADS anxiety scores in partners. Higher HADS depression scores of partners was associated with previous aortic surgery in the participants. Higher RDSQ score of partners was found to be associated with history of aortic dissection in the participants. The results of the univariable and multivariable analyses are displayed in online supplemental file 5 .

\section{In-depth interviews}

Eleven participants (six females, mean age 57 years) were interviewed: four had a low scores; four had intermediate and three had high scores on the HADS and the RDSQ; four had known genetic mutations (Loeys-Dietz syndrome $(n=3)$, Marfan syndrome $(n=1))$; five had familial TAD but no genetic diagnosis; three had already undergone aortic surgery, one had been accepted for surgery. Anxiety was not found to be a major topic in the interviews. Participants described a form of psychological distress related to having TAD and the risk of sudden events. Roughly, three groups could be distinguished, representing (almost) no psychological distress $(n=5)$; moderate psychological distress $(n=3)$ or prominent psychological distress $(n=3)$. Among these groups there was great variability in the degree their daily life, personality and professional career were affected. Several factors seemed to positively influence the level of psychological distress: disease-related communication with family members; level of the disease-related knowledge of the participant; evolution of the aortic disease (stable aortic diameter) and coping strategies such as expressing emotions and seeking emotional support. Factors that negatively influenced the level of distress were: physical complaints; sense of loss of identity due to the disease, including sports participation and choice of profession. Figure 4 was made to create an overview and shows which of these factors resulting from the coding tree were found to influence psychological distress the most. All participants had physical complaints such as thoracic pain, dyspnoea or tachycardia causing psychological distress. Sense of loss of identity was found to be a trigger in a smaller number of participants. Regular controls and stable aortic diameters had a great positive influence on psychological distress for the majority of participants. Knowledge about the disease seemed to decrease distress, although some participants expressed becoming more distressed after knowing what could happen. The unpredictable and uncertain course of the disease triggered a certain loss of control and increased psychological distress in almost all participants. Lifestyle restrictions on physical activity 
Aortic and vascular disease

Table 3 Scores of partners compared with participants and with the general population

\begin{tabular}{|c|c|c|c|c|c|}
\hline & $\begin{array}{l}\text { Partners } \\
(n=66)\end{array}$ & Participants $(n=261)$ & P value & General population & $P$ value \\
\hline \multicolumn{6}{|l|}{ RDSQ } \\
\hline Total score & $49.5(32.5-77.0)$ & $51.0(32.0-80.0)$ & 0.784 & & \\
\hline \multicolumn{6}{|l|}{ SF-36 } \\
\hline Physical Functioning & $76.1 \pm 25.2$ & $76.1 \pm 26.5$ & 0.991 & $83.0 \pm 22.8$ & 0.035 \\
\hline Role Physical & $65.6 \pm 41.1$ & $67.4 \pm 4.6$ & 0.748 & $76.4 \pm 36.3$ & 0.044 \\
\hline Bodily Pain & $74.1 \pm 25.4$ & $75.5 \pm 25.7$ & 0.707 & $74.9 \pm 23.4$ & 0.806 \\
\hline General Health & $55.8 \pm 21.8$ & $55.4 \pm 24.4$ & 0.902 & $70.7 \pm 20.7$ & $<0.001^{*}$ \\
\hline Vitality & $60.1 \pm 23.4$ & $59.0 \pm 25.1$ & 0.768 & $68.6 \pm 19.3$ & $0.006^{*}$ \\
\hline Social Functioning & $80.1 \pm 24.2$ & $79.2 \pm 25.8$ & 0.802 & $84.0 \pm 22.4$ & 0.216 \\
\hline Role Emotional & $84.7 \pm 31.4$ & $77.2 \pm 37.5$ & 0.108 & $82.3 \pm 32.9$ & 0.553 \\
\hline Mental health & $76.9 \pm 17.1$ & $75.2 \pm 19.4$ & 0.529 & $76.8 \pm 17.4$ & 0.957 \\
\hline PCS & $45.5 \pm 11.5$ & $46.4 \pm 11.5$ & 0.568 & & \\
\hline MCS & $50.1 \pm 9.7$ & $48.2 \pm 11.5$ & 0.255 & & \\
\hline \multicolumn{6}{|l|}{ HADS } \\
\hline Anxiety score & $4.0(2.0-6.0)$ & $4.0(2.0-7.0)$ & 0.554 & & \\
\hline Depression score & $1.0(0.0-4.0)$ & $2.0(1.0-5.0)$ & 0.158 & & \\
\hline Total score & $5.5(3.3-10.0)$ & $6.0(3.0-12.0)$ & 0.112 & & \\
\hline
\end{tabular}

Data are expressed as medians (IQR) when non-parametrically distributed. However, for comparison with the general population mean \pm SD was used.

*Significant after Bonferroni correction.

HADS, Hospital Anxiety and Depression Scale; MCS, Mental Component Summary; Participants, screening participants and patients with thoracic aortic disease; PCS, Physical Component Summary; RDSQ, Rotterdam Disease Specific Questionnaire; SF-36, Short Form 36.

and professional career were accepted by most of the participants. The majority $(n=9)$ stated that their level of distress increased shortly prior to regular check-ups of the aortic diameter, and decreased again when the results showed a stable aortic diameter. Coping strategies seemed to influence all factors mentioned above. A more detailed description of the in-depth interviews results can be found in online supplemental file 6 .

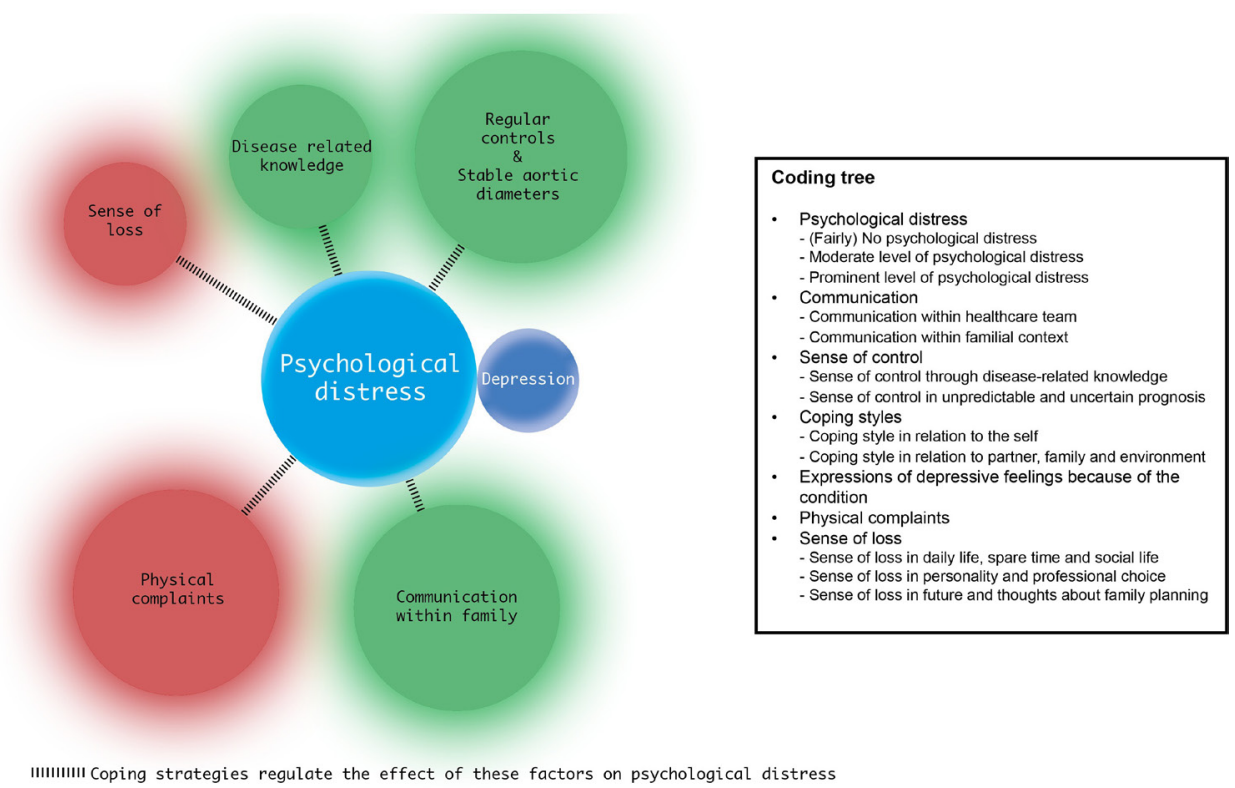

Figure 4 The influence on psychological distress of factors described in the coding tree. Red circle=negative influence on psychological distress; green circle=positive influence on psychological distress. The size of the circles corresponds with the amount of participants in whom psychological distress was triggered by this factor. Depression can result from a large amount of psychological distress. Patients' coping strategies regulate the effect of these factors. 


\section{DISCUSSION}

To our knowledge, this is the first study describing malefemale-specific aspects of HRQOL in both screening participants and patients with TAD using a mixed methods approach. Male and female patients with TAD reported clearly impaired HRQOL compared with the general population, while screening participants were affected to a lesser extent. Moreover, female and younger participants showed the lowest HRQOL scores. HRQOL in partners was also found to be suboptimal, although less impaired than in patients with TAD. Additionally, level of anxiety in patients with TAD was not found to be a major topic in the in-depth interviews, participants rather described a form of psychological distress.

In this study, patients with TAD were found to have decreased HRQOL, coping problems and psychological distress, similar to the findings of Olsson et al and Connors et $a .^{5}{ }^{18}$ Additionally, we found HRQOL in screening participants was impaired, although to a lesser extent. We hypothesised that the risk of having TAD would equally impact screening participants compared with patients with TAD. However, having an aortic diameter $\geq 40 \mathrm{~mm}$ clearly had a more profound impact on HRQOL. This does not seem to result in clinically relevant levels of anxiety or depression, since HADS scores of patients with TAD were comparable to the general population. As has been reported before, most of our participants accepted restrictions on physical activity and employment as "part of life'. 18

In the general population, HRQOL is known to decline with increasing age. ${ }^{12}$ We found a much less pronounced decline in patients with TAD. Presumably, the impact of having a serious disease with reduced life expectancy is more distinct at young age. In addition, we found less favourable scores, and more anxiety in females compared with males. This is also described in the general population $^{12}{ }^{19}$ and in patients with heart disease as well as chronic kidney disease, ${ }^{20}{ }^{21}$ but has not previously been reported in patients with TAD. ${ }^{6}$ Multiple studies reported these male-female differences were largely attributable to sociodemographic factors such as income, physical activity and marital status. ${ }^{22} 23$ This might also apply to our population, since employment status and physical activity were significantly different between males and females. However, this does not completely seem to explain malefemale difference in HRQOL. ${ }^{23}$ Another potential explanation might be that females experience their functional capacity and quality of life different from males, and this certainly needs more attention in future studies.

Partners' HRQOL was also found to be affected, although to a lesser extent, and seemed to be negatively influenced by factors related to aortic events and disease progression in the participants. HADS anxiety scores in partners were associated with ARB use of the participants. This may be due to the increased risk of aortic expansion and eventually dissection when hypertension persists. The need of antihypertensive medication might therefore cause more worries and anxiety in partners. The impact of TAD on partners has been underexposed, but might be very important, especially since interviewed participants reported that communication with their partners and family about the disease was an important factor influencing their level of psychological distress. Therefore, we believe the impact of the disease on the quality of life in partners should be assessed on a regular basis as it might impact the HRQOL of the patients. More research is needed to evaluate which factors are important and can be easily addressed. It is essential to involve partners in counselling and interventions for HRQOL improvement, and provide practical advice on coping with the disease for patients as well as their partners.

Goldfinger et al and Moon et al showed that daily life aspects such as employment or health insurance impacted HRQOL rather than disease-related factors. ${ }^{8} 24$ In contrast, we found mainly disease-related factors, such as a history of aortic dissection or surgery and the diameter of the aorta, to be associated with HRQOL. These factors emerged from results of both the quantitative and qualitative analyses. Potentially, this is due to the presence of a reliable social-security system in the Netherlands. Interviewed participants experienced the disease as a continuous threat, as was previously described in patients with abdominal aortic aneurysm. ${ }^{25}$ When looking into the RDSQ results in more detail, we noticed the highest scores were found on questions about heredity and surgery (online supplemental file 4), which indicates these factors warrant attention. Our findings emphasise the importance of expectation management and counselling regarding the disease course and treatment plan to the patient and their partner or family members, as well as providing clear and patient specific information about the disease and treatment options for shared decision making. Clinicians should be aware of HRQOL impairments, anxiety and depression in patients with TAD and their partners, and systematic screening is mandatory. Several factors were identified which might improve HRQOL: employment of constructive coping strategies such as expressing one's emotions, seeking emotional support, grieving loss of one's identity; and good communication within the family. When indicated, psychological support should be offered. More research is needed to evaluate the potential positive effect of psychological support and counselling in these patients.

\section{Limitations}

In this study, we used three (digital) questionnaires to measure self-reported quality of life. Two are wellknown validated questionnaires, the RDSQ questionnaire, however, was newly developed and used in one previous study so far. ${ }^{10}$ Its psychometric properties have to be further evaluated. Therefore, results of this questionnaire are to be interpreted with caution. The SF-36 is more suited to assess overall HRQOL and the HADS was used for detecting clinically relevant anxiety and depression. With the addition of the RDSQ, especially 
when combined with the results from the in-depth interviews, we were able to identify specific disease-related factors influencing HRQOL in this population. The overall response rate of patients participating in this study was $57 \%$, which is comparable to other (online) surveys. ${ }^{26}$ In order to provide insight into potential selection bias, the selection process is represented in figure 1 . Although some bias cannot be ruled out, we expect to have included a representative sample. The number of partners included was limited (25\%). Not all patients had a partner, but still this number is relatively low and better coverage of the partners is important for future research.

\section{CONCLUSIONS}

HRQOL of patients with TAD was found to be impaired, most significantly in younger females. Previous aortic surgery, aortic dissection and larger diameter of the aorta were found to be associated with impaired HRQOL. Partners' HRQOL was also reduced, warranting further attention. Patients with TAD and partners should be systematically screened for symptoms of depression and anxiety, and when indicated psychological support should be offered. Counselling patients to employ constructive coping strategies and good communication within the family might reduce psychological distress and improve HRQOL in this population.

\author{
Author affiliations \\ ${ }^{1}$ Cardiology, Erasmus Medical Center, Rotterdam, The Netherlands \\ ${ }^{2}$ Cardiology, Radboud University Medical Centre, Nijmegen, The Netherlands \\ ${ }^{3}$ Cardiothoracic Surgery, Erasmus Medical Center, Rotterdam, The Netherlands \\ ${ }^{4}$ Department of Child and Adolescent Psychiatry/Psychology, Erasmus Medical \\ Center, Rotterdam, The Netherlands \\ ${ }^{5}$ Research Institue of Child Development and Education, De Bascule-AMC, \\ University of Amsterdam, Amsterdam, The Netherlands \\ ${ }^{6}$ Paediatric Care, Erasmus Medical Center, Rotterdam, The Netherlands \\ ${ }^{7}$ Departement of Public Health and Primary Care, KU Leuven, Leuven, Belgium \\ ${ }^{8}$ Fonds Wetenschappelijk Onderzoek-Vlaanderen, Brussels, Belgium \\ ${ }^{9}$ Department of Nursing, UAntwerpen, Antwerp, Belgium
}

Contributors CGET and SD provided substantial contributions to the conception, design, data collection, data analysis, interpretation of the data and preparation of the manuscript. JWR-H supervised this project from its conception, including the study design, data analysis and interpretation and manuscript preparation. EG cosupervised the project on qualitative study design, data analysis and interpretation and manuscript preparation. These authors agree to be accountable for all aspects of the work in ensuring that questions related to the accuracy or integrity of any part of the work are appropriately investigated and resolved. LRB, ALG, RMK, AEvdB, JAAEC, EMWJU, RRLvK, JJMT and LMHR provided substantial contributions to the conception, design and drafting of this work, aided in revising the work critically for important intellectual content and provided final approval of the version to be published. All authors consented to the submission of this manuscript.

Funding This study was supported by ZonMW (project number: 849200014 ) and by an EBCN grant of the Erasmus MC (project number: 108600).

Competing interests None declared.

Patient consent for publication Not required.

Ethics approval This study was approved by the local ethics committee (METC Erasmus MC, MEC-2017-057), and was designed, performed and controlled in accordance with current local and international Good Clinical Practice guidelines. Written and signed informed consent was obtained from all participants. This study was designed and performed without patient involvement.

Provenance and peer review Not commissioned; externally peer reviewed.
Data availability statement All data relevant to the study are included in the article or uploaded as supplementary information. The data that support the findings of this study are available from the corresponding author (JWR-H) on reasonable request.

Open access This is an open access article distributed in accordance with the Creative Commons Attribution Non Commercial (CC BY-NC 4.0) license, which permits others to distribute, remix, adapt, build upon this work non-commercially, and license their derivative works on different terms, provided the original work is properly cited, appropriate credit is given, any changes made indicated, and the use is non-commercial. See: http://creativecommons.org/licenses/by-nc/4.0/.

ORCID iDs

Eva Goossens http://orcid.org/0000-0003-3421-5256

Jolien W Roos-Hesselink http://orcid.org/0000-0002-6770-3830

\section{REFERENCES}

1 Olsson C, Thelin S, Ståhle E, et al. Thoracic aortic aneurysm and dissection: increasing prevalence and improved outcomes reported in a nationwide population-based study of more than 14,000 cases from 1987 to 2002. Circulation 2006;114:2611-8.

2 Mehta RH, Suzuki T, Hagan PG, et al. Predicting death in patients with acute type A aortic dissection. Circulation 2002;105:200-6.

3 Albornoz G, Coady MA, Roberts M, et al. Familial thoracic aortic aneurysms and dissections--incidence, modes of inheritance, and phenotypic patterns. Ann Thorac Surg 2006;82:1400-5.

4 Erbel R, Aboyans V, Boileau C, et al. 2014 ESC guidelines on the diagnosis and treatment of aortic diseases: document covering acute and chronic aortic diseases of the thoracic and abdominal aorta of the adult. The task force for the diagnosis and treatment of aortic diseases of the European Society of cardiology (ESC). Eur Heart J 2014;35:2873-926.

5 Olsson C, Franco-Cereceda A. Health-Related quality of life in thoracic aortic disease: Part I. cases managed Non-operatively. Aorta 2013;1:153-61.

6 Velvin G, Wilhelmsen JE, Johansen $\mathrm{H}$, et al. Systematic review of quality of life in persons with hereditary thoracic aortic aneurysm and dissection diagnoses. Clin Genet 2019;95:661-76.

7 Rand-Hendriksen S, Johansen H, Semb SO, et al. Health-Related quality of life in Marfan syndrome: a cross-sectional study of short form 36 in 84 adults with a verified diagnosis. Genet Med 2010;12:517-24.

8 Goldfinger JZ, Preiss LR, Devereux RB, et al. Marfan syndrome and quality of life in the GenTAC registry. J Am Coll Cardiol 2017;69:2821-30.

9 Fusar-Poli P, Klersy C, Stramesi F, et al. Determinants of quality of life in Marfan syndrome. Psychosomatics 2008;49:243-8.

10 Bons LR, van den Hoven AT, Damirchi AE, et al. Psychological wellbeing in patients with aneurysms-osteoarthritis syndrome. Am J Med Genet A 2019;179:1491-7.

11 Du Bois D, Du Bois EF. A formula to estimate the approximate surface area if height and weight be known. 1916. Nutrition 1989;5:303-11. discussion 12-3.

12 Aaronson NK, Muller M, Cohen PD, et al. Translation, validation, and norming of the Dutch language version of the SF-36 health survey in community and chronic disease populations. J Clin Epidemiol 1998;51:1055-68.

13 Ware JE, Sherbourne CD. The mos 36-item short-form health survey (SF-36). I. conceptual framework and item selection. Med Care 1992;30:473-83

14 Spinhoven P, Ormel J, Sloekers PP, et al. A validation study of the hospital anxiety and depression scale (HADS) in different groups of Dutch subjects. Psychol Med 1997;27:363-70.

15 Bjelland I, Dahl AA, Haug TT, et al. The validity of the hospital anxiety and depression scale. An updated literature review. J Psychosom Res 2002;52:69-77.

16 Utens B R-H. Rotterdam disease specific questionnaire for thoracic aortic disease (TAD. Erasmus MC, 2017.

17 Boeije H. Analyseren in kwalitatief Onderzoek, denken en doen. Boom, 2019.

18 Connors E, Jeremy RW, Fisher A, et al. Adjustment and coping mechanisms for individuals with genetic aortic disorders. Heart Lung Circ 2015;24:1193-202.

19 McLean CP, Asnaani A, Litz BT, et al. Gender differences in anxiety disorders: prevalence, course of illness, comorbidity and burden of illness. J Psychiatr Res 2011;45:1027-35.

20 Huber A, Oldridge N, Höfer S. International SF-36 reference values in patients with ischemic heart disease. Qual Life Res 2016;25:2787-98. 
21 Zimbudzi E, Lo C, Ranasinha S, et al. Predictors of health-related quality of life in patients with co-morbid diabetes and chronic kidney disease. PLoS One 2016;11:e0168491.

22 Cherepanov D, Palta M, Fryback DG, et al. Gender differences in health-related quality-of-life are partly explained by sociodemographic and socioeconomic variation between adult men and women in the US: evidence from four us nationally representative data sets. Qual Life Res 2010;19:1115-24.

23 Hajian-Tilaki K, Heidari B, Hajian-Tilaki A. Are gender differences in health-related quality of life attributable to sociodemographic characteristics and chronic disease conditions in elderly people? Int J Prev Med 2017;8:95.
24 Moon JR, Cho YA, Huh J, et al. Structural equation modeling of the quality of life for patients with Marfan syndrome. Health Qual Life Outcomes 2016;14:83.

25 Berterö C, Carlsson P, Lundgren F. Screening for abdominal aortic aneurysm, a one-year follow up: an interview study. J Vasc Nurs 2010;28:97-101.

26 Ebert JF, Huibers L, Christensen B, et al. Paper- or web-based questionnaire invitations as a method for data collection: crosssectional comparative study of differences in response rate, completeness of data, and financial cost. J Med Internet Res 2018;20:e24. 